\title{
Road Travel Time Information on VMS and Traffic Congestion
}

\author{
Brigitte Cambon de Lavalette*, Charles Tijus**, Sébastien Poitrenaud ${ }^{* *}$, Christine Leproux** \\ *LPC-INRETS France \\ **Laboratoire Cognition et Usages, Université de Paris 8, France
}

\begin{abstract}
Some years ago, progress in road telematics had made it possible to introduce time travel information in real time on VMS devices. Such information can lead road users to avoid congested areas as well as to waiting in a better condition for the end of the congestion. In a way, they increase congestion, in another way, they reduce it. Our research aimed at explaining the reasons why some people behave in a way and another one in. We assumed that travel time messages may be interpreted from several points of view, depending on driver knowledges in memory, experience and will, and as a result, the decision taken.

An enquiry on a group of drivers aimed at analysing mental activity involved in decision taking process will be presented.
\end{abstract}

\section{INTRODUCTION}

Traffic saturation in urban areas is a never ending problem for traffic managers. Though urban expressways were originally conceived for easing transit, little by little they have also become the scenes of daily congestions. This state of things is not without consequences for the quality of life in the cities, on account of the loss of time involved or even the irritation expressway users may feel when they are trapped in traffic jams.

Progress in road telematics has introduced variable message signs (VMS's) which should constitute a precious aid to traffic engineering. It can provide a way in regulating traffic stream as well as to drivers who wish to know how long it needs to reach their destination. These are devices designed to improve the flow of traffic according to a self-regulating process: the driving time between two points is displayed in real time, drivers can evaluate the state of traffic and may choose another less encumbered route if need be.
However, the semantics of VMS messages are clearly different from traditional signposts which do not provide for announcing transitory events in real time. How do drivers interpret these messages, what are they lead to decise from them?

At the beginning, when travel times appear on VMS in the road network, they were supposed to be used to avoid congested areas. In fact, according to the opinion polls commissioned by the transportation authorities, although drivers are for the most part $(98 \%)$, glad to benefit from this new service, most of the time, they are no more likely to change their itinerary when the expressway is crowded (Ref. 1). In other words, the VMS's are probably useful to users because they have expressed their satisfaction with them, but the use they seem to make of them is seemingly not the use for which they were intended. As a result, travel time information affects traffic congestion at the opposite of expected traffic state.

Our research was aimed at understand the reasons which in the driver mental process can explain such an effect. At the same time, we observed the road user needs according to time travel information.

The device which the use we observed is put on an expressway that circles Paris. It is a ring road $37 \mathrm{~km}$. long, called the "Boulevard Périphérique", that connects the 32 "Gates" into the city. The VMS's stationed approximately every 500 metres indicate two destinations (which are "gateways" into the city), followed by the driving time it will take to get there (see figure 1). The system which produces these messages functions as follows. Sensors embedded in the carriageway calculate several variables in real time: traffic flow, speed and a representation of traffic density. The sensors are spaced approximately 500 metres apart, as are the signposts; and measurements are taken every minute.

\section{HOW DO DRIVERS INTERPRET TRAVEL TIME MESSAGES ?}

Traffic measurements and observations of behaviour have already been carried out to evaluate the impact of variable message signs on driver behaviour (2). The results reveal reactions which are difficult to 
interpret within the generally adopted framework of driver rationality. We therefore need to understand the mental process involved in reading the messages before the driver takes any decisions and the types of

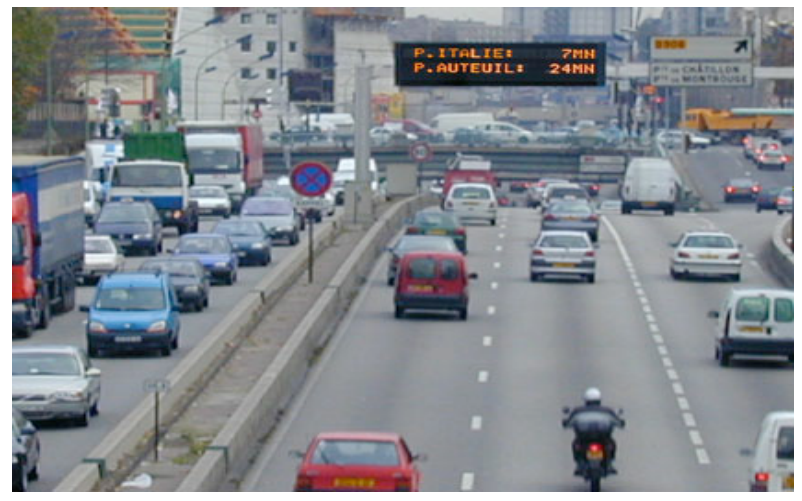

Figure 1. Variable Message Signs device on the expressway around Paris (Photo. INRETS)

reasoning drivers use when deciding whether to continue on or to leave a saturated route. In view of the mental process involved, the decisional process that precedes decision making may involve several motives and inferences that are probably more complex than those which are immediately apparent. Two types of questions are considered in turn: first, what knowledge is involved in interpreting messages and determines the meaning ascribed to them, and second what intentions may inform the decision in favour of one or other alternative, namely keeping to the same route or changing it.

\subsection{KNOWLEDGE INVOLVED IN MESSAGE UNDERSTANDING}

Obviously, one factor involved in message understanding is knowledge required to interpret what it is meaning at. Time travel messages are alphanumerical and brief. They state a location and a duration, which means that the travel time between the sign and the location which is downstream on the same road is currently the number of minutes indicated on the device. The elliptical nature of the messages assumes that drivers already possess a certain amount of knowledge, if only topographical, about the area and are also able to represent and process this knowledge, which implies diversified mental process that uses processes whose paths must be mapped out in order for the outcome to be understandable. Then, knowledge refer to all the memorized information involved in the interpretation of the message but also some properties of this knowledge, for example the reliability of travel time measurement process. It is easy to see that it is important to trust the reliability of the measurement device in order to base an action on it. The action drivers decide to take in response to messages depends on how reliable they think they are. Drivers must therefore understand that the displayed journey time is the journey time for the journey they will make. The main value of the time displayed on variable message signs is that it is the result of a real time assessment and gives an instantaneous picture of the situation at the moment in question. Drivers need to understand that the assessment is output by a system that collects data on traffic conditions at the instant in question. It is therefore necessary to have constructed a mental representation of a system that is able to produce reliable current traffic data.

We can identify various types of knowledge in this context. For example, when the message states a travel time longer than normal, a driver may infer the presence of congestion. However, a driver who does not know the normal time travel is not able to interpret the information correctly because he or she lacks an adequate mental model. A driver who knows the locality and the normal journey time but does not know an alternative route has an accurate representation of the content of the message but does not possess the appropriate response in order to modify his or her behaviour. Lastly, a driver who does not have a satisfactory mental model of the operation of the system can interpret one of the items of information in a way other than that which is intended. For exemple, in Paris, where this research took place, the message "Porte de Bercy 5 min » could thus be taken as meaning «I passed the Porte de Bercy 5 min ago " or "The displayed journey time is short because they want motorists to use this route rather than another ". And then, if he ignores how the message gets to the the sign, he can assume that the real time is now different.

Briefly, knowledge we considered were the following :

- Knowledge concerning the typonomy and typology of the locations.

- Knowledge of usual time travel for the destination mentioned 
- Knowledge of alternative routes (mental map)

- Trust in the reliability of the messages

\subsection{DRIVER INTENTION CONCERNING SPEEDNESS}

Traffic counts have revealed that time travel information does not lead to any specific route changes. Drivers who are faced with the choice of leaving a saturated motorway in order to use a freely flowing alternative route prefer to stay on the saturated road, even though they know how much time they will lose as a result. This suggests that the intention of saving travel time, which is very often ascribed to drivers, is not all that dominant. Knowledge is certainly necessary, but it in not enough to induce action. It seems to us that for a driver to use his knowledge, the desire to reach his destination as rapidly as possible must be accompanied by willingness and a capability to go by another route.

The implicit model (3) of the driver which is designed into the VMS system,", is that driving is a kind of "wanting to save time", to go as quickly as possible, faster than one can go by public transportation for example. But the extent to which this assumption is valid is not known, despite the fact that it is a commonly held belief. It seems self-evident that the more a person wants to reach his destination as quickly as possible, the more he will avoid heavy traffic. There is a need to verify that the subjects questioned on this point, aside from what they feel when they are actually in a traffic jam, really do have the intention of "saving time". It is then easy to see why it is important to verify this point with the subjects chosen.

It was apparent that, at an earlier stage than individual knowledge about the messages, drivers' intentions with regard to organizing their trips should also play a part in the decisions they take: what are their priorities when making a journey? We have considered two types of intentions:

- preserving one's comfort : keeping current action, avoiding to searchand to reach for another route

- reaching one's destination as rapidly as possible and saving time

\subsection{HYPOTHESIS}

Then, it could be assumed several categories of users. Here is an example: the driver's intention is to complete the journey quickly; with regard to knowledge the driver knows the location of the places, the usual journey times, but does not know an alternative route; with regard to reliability, the driver trusts the message. Another possible category could be: the driver's intention is to preserve his or her comfort; with regard to knowledge the driver knows the location of the places, the usual journey times and an alternative route; with regard to reliability the driver trusts the information.

Analysis of the inferences (4) that are generated by the messages is of interest on the following grounds: the driver's behaviour is not seen as the result of a single internal variable but as the outcome of a complex process made up of several successive analyses. On the basis of intentional forms and knowledge we can develop a taxonomy of the practices generated by messages. This is relevant not only to the regulation of transport but also to the more general attempt to find processes for analyzing the rationality of action.

To model this behaviour we have used Stone's model [5], which is based on knowledge networks. This establishes a taxonomy of the practices generated by the messages, which takes account of the user's knowledge of the system and message content (fig.2).. 


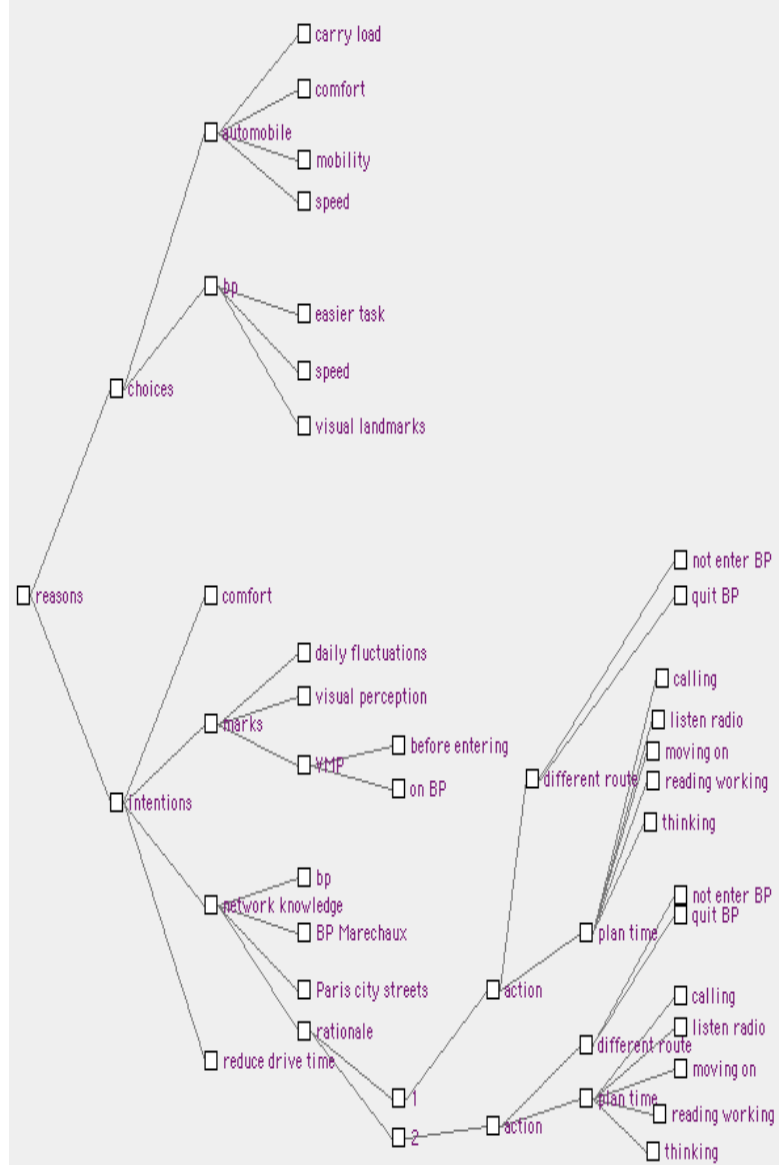

Figure 2. The whole set of properties that can define drivers as being a decomposition tree of actions, knowledge about routes, intention and motivs of choice for using automobile and for taking the the expressway .

\section{METHODOLOGY AND RESULTS}

The participants were told that they were being interviewed in order to obtain a description of the route they take to get to work (6). The interviews with the driver-participants were conducted in such a way as to gather information on the following themes.

1. The choice of the automobile over other means of transportation, the importance of "time pressure" in this choice and the reasons for using the boulevard peripherique (BP).
2. An account of the most recent actual itinerary taken, the objective of which was to gather the route the driver actually takes and the actual decisions he makes.

3. Evocation of the different routes taken, in order to get the participant to spontaneously reveal the importance of VMS messages on driving time in choice of an itinerary.

4. A description of the way the VMS system works.

Figure 2 presents Stone's formalization of the links between the successive states entailed in the decision to stay on a route or to abandon it when the VMS system announces a seemingly excessive driving time. The arborescent structure represents the alternative motives which compose the plan of action: the intention inferred from the reasons for choosing to go by automobile, to take the BP; knowledge about the configuration of the system, the grounds on which drivers make their decision; the inferences they draw from the messages displayed on the VMS before getting on to the BP (action 1), then when they are already on the BP and a traffic jam suddenly forms (action 2). In both cases, the decision is either to change itineraries so as to avoid the jam, or to rest in traffic and think of something to do while waiting.

When there are traffic jams, what do drivers decide to do? According to results, we ascertained that there are three different types of behavior regarding this decision : some (group 1, 7 participants, $23 \%$ ) change itineraries whenever traffic is slow, others (group 2, 18 participants, $60 \%$ ) stay in traffic no matter what (even in the worst traffic jams), and still others (group 3, 5 participants, $16 \%$ ) will enter or stay in slower traffic providing that the driving times displayed by the VMS's are not excessive. 
The first group (23\%) is composed of the drivers whose primary goal is to reach their destination as quickly as possible. These participants seem to be familiar with alternative routes: 4 use secondary routes inside Paris (city streets). Because they know alternative routes, they can quit the BP when traffic is slow. All of them use various sources of traffic information in addition to the VMS system before deciding on the route to take. All except one change itineraries if when they arrive at the BP, the VMS's indicate a traffic jam. If a traffic jam forms while they are already on the BP, they get off. If the driving time the VMS displays when they arrive at the BP seems excessive to them, they decide on an alternative route and do not enter traffic on the BP. Their decision to desist is facilitated by the fact that in general they have a working knowledge of alternative routes, "emergency" routes to which they turn in case of traffic jams. Thus, driving time information helps them avoid crowded routes.

In contrast to the first group are the group of participants $(60 \%)$ on whom the system seems to have no effect: they do not change itineraries when the driving time displayed suggests there are traffic jams. They seem to accept traffic jams very patiently and are the most numerous among our participants. They never change their itinerary regardless of what is announced on the VMS at the entry to the BP or while they are on it, preferring to make themselves as comfortable as possible and wait out the time. Most of them know of few alternative routes and do not try to find out what these may be. Users in this category show little readiness for finding alternatives to the BP, their knowledge of the network of roads outside of the BP is limited, and this limits their ability to choose an alternative route. However, even after years of driving, they do not seem to want to venture off their familiar itinerary, which they seem attached to. Nonetheless, they also try to save time; some of them even leave earlier to avoid traffic jams. The information on driving times reinforces their conviction that "in any case, it's the same on all the roads...", and that there is little use in attempting to change routes.

Finally, we ascertained that there was a third group of participants (16\%) who show behaviour somewhere in between the two behaviours described above. This category is composed of participants with mixed goals: some prefer the comfort of the ride, others emphasise speed. Unlike the previous group, drivers in this group do not get on the BP when a traffic jam had been announced. However, if a jam forms when they are already on it, they do not get off. They rely on the driving times displayed to make their decisions. All of them have a working knowledge of alternative routes, and all of them also use the time spent in slower traffic to engage in other activities. It is very likely that the messages on driving time reduce the stress of drivers in this group. As one of them says, If I see that the traffic jam is going to last for a while and that... oh well, too bad, I'm just going to have to wait it out. Like the second group of drivers, reaching their destinations as quickly as possible is not always what most interests them, however, the reason they give for taking the BP is that it is faster, and in this they resemble the first group. They will not get onto the BP if traffic is backed up but nonetheless, like participants in the second group, they accept having to lose some time, though this acceptance is not unconditional and remains within limits that they can usually specify. They know the road network well and can change itineraries when need be. They use VMS's either to avoid getting onto a jammed BP (as indicated by a VMS or from what they can see) or, if a traffic jam forms when they are already on it, the information helps them to plan how to use the time spent waiting.

The assumption that drivers wish to reach their destinations as quickly as possible is not valid for all 
drivers: only $23 \%$ of participants emphasised wanting to reach their destination as quickly as possible and $60 \%$ emphasised the comfort of driving. The whole set of results show that the implicit model of the user which is a necessary component of the self-regulating system does not correspond to all users. On the contrary, there are users who settle down comfortably into traffic jams: they make telephone calls, listen to music, etc. As one of them states very clearly :... I like the intimacy of my car... it's like a small living room in which I can listen to whatever music I feel like hearing. I don't have to stand contact with other people, I can read the paper, there are many things you can do in a car... Being stuck in traffic is thus not necessarily something that all drivers seek to avoid, since they know how long they will be. It is not congestion that would make trouble to the drivers, but to be keep in the dark about its duration. This "comfort" category of user does not correspond to the usual user model.

\section{CONCLUSION}

When a driver reads the driving time displayed on a variable message signpost (VMS) and the driving time indicates there are traffic jams ahead, two courses of action are open to him: he can either abandon the road for another he believes will be quicker or stick to his itinerary. Our approach was designed to reveal the modalities of this decision to stick to an itinerary or to change it, depending on the information delivered by the VMS's (for example in Paris, Porte d'Auteuil, 24 minutes), by studying interpretations of the contents of these messages in terms of how the system functions (where the data is thought to come from), and the contextual processing of the displayed data (the driver's task, how the traffic looks) and finally decision making processes, but also the implicit model of the user for whom the messages are conceived and displayed.

\section{REFERENCES}

(1) Cohen, S., \& Hadj-Salem, H. (1996) Évaluation comparative des affichages bouchons et temps de parcours. L'expérience de la ville de Paris. Recherche, Transports, Sécurité, 51, 29-39.

(2) Jardin, P., \& Laterrasse, J. (1998) Méthode d'analyse de l'impact des informations dynamiques des panneaux à message variables sur le comportement des usagers franciliens. In : Actes du Congrès International de l'ATEC "Déplacements : l'ère de la gestion. (pp 159170). Paris : Presses des Ponts et Chaussées.

(3) Tijus, C., Cambon de Lavalette, B., Poitrenaud, S. and Leproux, C., 2003, L'interaction autorégulatrice entre dispositif et utilisateur: une modélisation des inférences sur les durées du parcours routier, Le Travail Humain 66:23-44.

(4) Tauber, M. (1988). On mental models and the user interface. In G.C. Van Der Veer, T.R.G. Green, J.M. Hoc, \& D.M. Murray (Eds.), In Working with Computers: Theory versus Outcome (pp. 89-121). Londres: Academic Press.

(5) Poitrenaud, S. (1995). The Procope Semantic Network: an alternative to action grammars. International Journal of Human-Computer Studies, 42, 31-69.

(6) Flanagan, J. C. (1954). The critical incident technique. Psychological Bulletin, 51, 327-359. 